\title{
ELECTRIC FIELD GRADIENTS FOR SPIN 3/2 NUCLEI FROM NQR MEASUREMENTS
}

\author{
K. V. Raman AND P. T. NARASIMHAN $\dagger$ \\ Department of Chemistry, \\ Indian Institute of Technology, \\ Kanpur-16, India
}

\begin{abstract}
Due to the $\pm m$ degeneracy in the absence of a magnetic field, nuclear quadrupole resonance (nqr) measurements of electric field gradients for spin $\frac{3}{2}$ nuclei are incomplete without Zeeman effect studies if the asymmetry parameter $\eta \neq 0$. A large number of Zeeman studies have been carried out using single crystal specimens making use of either the zero-splitting locus method or Rehn's method. The powder method originated by Morino and Toyama provides a very convenient procedure for obtaining asymmetry parameter values for spin $\frac{3}{2}$ nuclei, and thus the electric field gradients, and is especially valuable when single crystal specimens are difficult to obtain. No doubt, measurements on single crystal specimens also provide information on the orientation of fieldgradient axes. In spite of the loss of such information the powder method has considerable attraction.

The equivalence of the results obtained by the powder method and the single crystal measurements was originally demonstrated by Morino and Toyama in two systems, namely, $p$-dichlorobenzene and cyanuril chloride. With this demonstration it may be thought that whether one obtains the electric field gradient data from a single crystal specimen or a powder specimen the results should be the same. In this paper the available results on asymmetry parameter measurements for two spin $\frac{3}{2}$ nuclei, namely, ${ }^{35} \mathrm{Cl}$ and ${ }^{71} \mathrm{Ga}$ in powder specimens, are presented and compared with single crystal data wherever available. It is found that except in two cases, namely mercuric chloride and gallium trichloride, the powder results and single crystal results agree very well. Factors that might contribute to the differences in the asymmetry parameter values obtained in the two situations are discussed.
\end{abstract}

\section{INTRODUCTION}

Due to the $\pm m$ degeneracy in the absence of a magnetic field pure quadrupole resonance measurements for spin $\frac{3}{2}$ nuclei are incomplete without Zeeman effect studies if the electric field gradients $\left(V_{x x}, V_{y y}, V_{z z}\right)$ are not axially symmetric, i.e. $V_{x x} \neq V_{y y}$. A large number of Zeeman studies of the asymmetry parameter $\eta=\left(V_{x x}-V_{y y}\right) / V_{z z}$ have been carried out with single crystal specimens making use of either the zero-splitting locus method ${ }^{1}$ or Rehn's

$\uparrow$ To whom all correspondence should be addressed. 
frequency-field method ${ }^{2}$. The powder method developed by Morino and Toyama $^{3}$ provides a very convenient procedure for obtaining asymmetry parameter values for spin $\frac{3}{2}$ nuclei and thus the electric field gradients. In spite of the fact that the powder method cannot provide information about the orientation of the field gradient axes, the method has considerable attraction. The experimental procedure used in this method is simple and it is very valuable for substances with low melting points. The equivalence of the results obtained by the powder method and the single crystal method was originally demonstrated by Morino and Toyama in two systems, namely, $p$-dichlorobenzene and cyanuril chloride. With this observation it may be thought that whether one obtains the electric field gradient data from the single crystals or powder specimens the results should be the same. In this paper available results on $\eta$ measurements for two spin $\frac{3}{2}$ nuclei, namely, ${ }^{35} \mathrm{Cl}$ and ${ }^{71} \mathrm{Ga}$, from powder data are presented and compared with single crystal data wherever available. It is found that except in two cases, namely mercuric chloride 4 and gallium trichloride ${ }^{5}$, the powder results and single crystal results agree very well. Factors that might contribute to the differences in the $\eta$ values obtained from powder specimens and single crystals are discussed. The value of computer-simulated theoretical Zeeman spectra for polycrystalline materials, especially in systems where closely spaced lines exist, is illustrated. A short review of the available methods for obtaining $\eta$ values for $I=\frac{3}{2}$ nuclei is presented in section 2 . The merits and demerits of the powder method are also pointed out there. In section 3 all results so far obtained for $I=\frac{3}{2}$ nuclei by the powder method are listed and compared with single crystal data wherever available. In section 4 the utility of computer simulation of spectra for the Zeeman study of powder specimens is illustrated using boron trichloride and $p$-dichlorobenzene as examples. In section 5 factors that might contribute to the differences in the $\eta$ values obtained using single crystal specimens and powder specimens are discussed.

\section{METHODS FOR DETERMINATION OF $\eta$ FOR $I=\frac{3}{2}$ CASE}

\section{(a) Methods using single crystals}

There are two well-known methods that have been extensively utilized in the determination of the asymmetry parameter $\eta$ for $I=\frac{3}{2}$ nuclei. These are the zero-splitting locus method ${ }^{1}$ and the frequency-field method ${ }^{2}$. Due to the fact that the energy levels in the presence of a magnetic field can be analytically expressed for the $I=\frac{3}{2}$ case with $\eta \neq 0$, the observed transitions in the case of single crystal specimens can be directly related to the asymmetry parameter and orientation of the electric field gradient axes. In particular, out of the four possible transitions, the two 'inner' lines can be made to coincide by suitable crystal orientation in the static magnetic field, $H$. The if field, $H_{1}$ is always kept at right angles to $H$. With $\theta$ and $\phi$ the angles which $H$ makes with $Z$ and $X$ axes respectively of the electric field gradients the condition for the coalescence of the inner pairs is

$$
\sin ^{2} \theta=\frac{2}{3-\eta \cos 2 \phi}
$$

In order to obtain $\eta$ accurately the method of least squares can be employed ${ }^{1}$. 
A computer program for carrying out the calculations utilizing the raw experimental data has also been outlined ${ }^{6}$. Experimentally, the most important measurements are the angle measurements in this method. The precision with which one can orient the crystal in the magnetic field determines largely the accuracy of $\eta$. Several workers ${ }^{7-9}$ have designed goniometer assemblies specifically for this purpose. The zero-splitting locus method is also referred to as the geometrical method. In the alternative method, namely the frequencyfield method of Rehn ${ }^{2}$, the separation between the 'inner' and 'outer' lines is used to obtain $\eta$ (with the magnetic field $H$ perpendicular to the $Z$ axis of the electric field gradient). $H_{1}$ is still kept perpendicular to $H$. In order to orient the crystal with $H$ perpendicular to the $Z$ axis of the electric field gradient it is necessary to carry out preliminary nqr investigations. The final determination of $\eta$ can be made by simply measuring the frequency difference between the inner and outer lines with $H$ perpendicular to the $Z$ axis. Due to the fact that a frequency measurement is involved, the accuracy of $\eta$ values obtained by this method is quite high ${ }^{10}$. Values of $H$ used range from a few gauss to a few hundred gauss.

\section{(b) The powder method}

In this method one determines the asymmetry parameter $\eta$ from a powder specimen placed in a weak magnetic field $H$ with the radio frequency field $H_{1}$ along $H$. The theory has been developed by Morino and Toyama ${ }^{3}$ who have shown that in the above situation the envelope of the Zeeman nqr absorption spectrum has two pairs of sharp peaks at $\pm(1 \pm \eta) v_{H}$ and one pair of sharp minima at $\pm v_{H}$, where $v_{H}$ is the Larmor frequency in the applied field $\left(v_{H}=\gamma H / 2 \pi ; \gamma=\right.$ gyromagnetic ratio). For the determination of $\eta$, it is necessary to measure the separation of each pair of adjacent deflections corresponding to $2 \eta v_{H}$ as defined in Figure 1. Due to the original linewidth these deflections are broadened and their locations may become somewhat

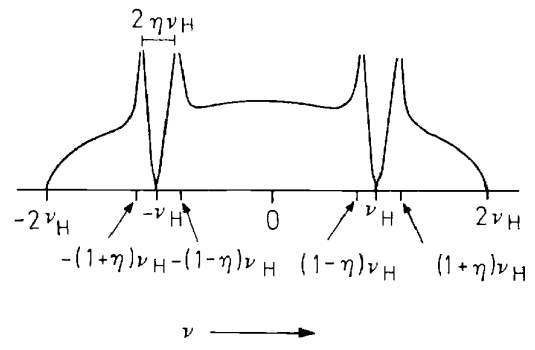

Figure 1. Predicted envelope shape for $I=\frac{3}{2}$ with the magnetic field $H_{1} \| H$ for $\eta \neq 0$ when the original linewidth is neglected (after Morino and Toyama ${ }^{3}$.)

uncertain. The determination of the points corresponding to $\pm(1 \pm \eta) v_{H}$ from the observed curves is therefore not easy. However, Morino and Toy$\mathrm{ama}^{3}$ have shown that an estimate of $\eta$ can be obtained by extrapolating the quantities $\delta_{i} / 2 v_{H}$ to infinite field strength, where $\delta_{1}, \delta_{2}$ and $\delta_{3}$ are as defined in Figure 2. The main difficulty in the powder method is that limitations on the maximum usable value of the magnetic field are imposed when the 


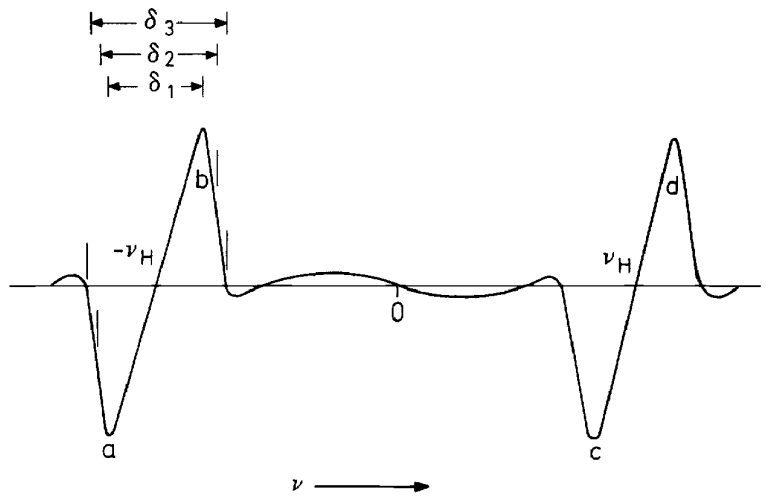

Figure 2. Derivative of the envelope shape for finite linewidth $\left(H_{1} \| H\right)$ (after Morino and Toyama ${ }^{3}$.

intensity of the Zeeman spectrum drops below noise level and when neighbouring resonance lines, if present, begin to overlap. Super-regenerative oscillators ${ }^{11}$ are used in order to get a high $S / N$ ratio. The difficulty encountered due to overlap between the unsuppressed side-band responses of self-

Table 1. Summary of asymmetry parameter values for spin $\frac{3}{2}$ nuclei using a powder specimen and comparison with single crystal data

\begin{tabular}{|c|c|c|c|c|c|}
\hline \multirow{3}{*}{$\begin{array}{l}\text { Compound } \\
\text { Antimony trichloride }\end{array}$} & \multirow{3}{*}{$\begin{array}{l}\text { Site } \\
{ }^{35} \mathrm{Cl}(\mathrm{I})\end{array}$} & \multirow{3}{*}{$\begin{array}{c}\begin{array}{c}\text { Frequency } \\
(\mathrm{MHz}) \text { at } 27^{\circ} \mathrm{C}\end{array} \\
19.1749\end{array}$} & \multicolumn{3}{|c|}{ Asymmetry parameter $(\eta)$} \\
\hline & & & \multicolumn{2}{|c|}{ Single crystal } & \multirow{2}{*}{$\frac{\text { Powder }}{0.16^{*, c}}$} \\
\hline & & & $0.153^{a}$ & $0.155^{b}$ & \\
\hline & ${ }^{35} \mathrm{Cl}(\mathrm{II})$ & 20.4074 & 0.057 & 0.0 & 0.06 \\
\hline \multirow[t]{4}{*}{ Gallium trichloride } & ${ }^{71} \mathrm{Ga}$ & 18.3150 & \multicolumn{2}{|c|}{$0.867^{d}$} & $0.26^{*, e}$ \\
\hline & ${ }^{35} \mathrm{Cl}(\mathrm{I})$ & 20.3020 & \multicolumn{2}{|c|}{0.034} & 0.63 \\
\hline & ${ }^{35} \mathrm{Cl}(\mathrm{II})$ & 19.2042 & \multicolumn{2}{|c|}{0.089} & 0.59 \\
\hline & ${ }^{35} \mathrm{Cl}(\mathrm{III})$ & 14.8118 & \multicolumn{2}{|c|}{0.473} & 0.45 \\
\hline \multirow[t]{2}{*}{ Chloranil } & ${ }^{35} \mathrm{Cl}(\mathrm{I})$ & 36.79 & \multirow{2}{*}{\multicolumn{2}{|c|}{$\begin{array}{l}0.21^{f} \\
0.21\end{array}$}} & \\
\hline & ${ }^{35} \mathrm{Cl}(\mathrm{II})$ & 36.86 & & & $0.196^{*, g}$ \\
\hline \multirow[t]{2}{*}{$p$-Dichlorobenzene } & ${ }^{35} \mathrm{Cl}$ & 3425 & \multirow{2}{*}{$\begin{array}{l}0.08^{h} \\
0.075^{k, \dagger}\end{array}$} & $0.05^{j}$ & $0,07 \|++, M$ \\
\hline & $\mathrm{Cl}$ & 34.25 & & $0.067^{l}$ & $0.07 \|, T^{\top} T^{-m e t}$ \\
\hline \multirow[t]{2}{*}{ Cyanuril chloride } & ${ }^{35} \mathrm{Cl}(\mathrm{I})$ & 36.300 & \multirow{2}{*}{$\begin{array}{l}0.23^{n} \\
0.26\end{array}$} & $0.24^{p}$ & $0.22 \|, m$ \\
\hline & ${ }^{35} \mathrm{Cl}(\mathrm{II})$ & 36.317 & & 0.24 & $0.23 \|$ \\
\hline Chlorine molecule & ${ }^{35} \mathrm{Cl}$ & $\begin{array}{l}54.248 \\
\text { (at } 77 \mathrm{~K} \text { ) }\end{array}$ & \multicolumn{2}{|c|}{ - } & $0.038^{\prime \prime}$ \\
\hline $\begin{array}{l}\text { Trichlorocyclopropenium } \\
\text { ion }\end{array}$ & ${ }^{35} \mathrm{Cl}$ & 39.325 & \multicolumn{2}{|c|}{ - } & $0.35 \|^{\prime s}$ \\
\hline \multirow[t]{2}{*}{ Mercuric chloride } & ${ }^{35} \mathrm{Cl}(\mathrm{I})$ & 22.2400 & \multirow{2}{*}{$\begin{array}{l}0.087^{t} \\
0.085\end{array}$} & $0.39^{u}$ & $0.30 \|^{* v}$ \\
\hline & ${ }^{35} \mathrm{Cl}(\mathrm{II})$ & 22.0578 & & 0.70 & $0.28 \|$ \\
\hline Silicon tetrachloride & ${ }^{35} \mathrm{Cl}$ & $\begin{array}{l}20.273 \\
(77 \mathrm{~K})\end{array}$ & \multicolumn{2}{|c|}{ - } & $0.45 \|^{x}$ \\
\hline Germanium tetrachloride & ${ }^{35} \mathrm{Cl}$ & $\begin{array}{l}25.451 \\
(77 \mathrm{~K})\end{array}$ & \multicolumn{2}{|c|}{ - } & $0.35 \|^{, x}$ \\
\hline Tin tetrachloride & ${ }^{35} \mathrm{Cl}$ & $\begin{array}{l}23.719 \\
(77 \mathrm{~K})\end{array}$ & \multicolumn{2}{|c|}{-} & $0.25 \|^{; x}$ \\
\hline
\end{tabular}


ASYMMETRY PARAMETERS FOR SPIN 3/2 NUCLEI

Table 1.--continued.

\begin{tabular}{|c|c|c|c|c|}
\hline \multirow{2}{*}{ Compound } & \multirow{2}{*}{ Site } & \multirow{2}{*}{$\begin{array}{c}\text { Frequency } \\
(\mathrm{MHz}) \text { at } 27^{\circ} \mathrm{C}\end{array}$} & \multicolumn{2}{|c|}{ Asymmetry parameter $(\eta)$} \\
\hline & & & Single crystal & Powder \\
\hline Carbon tetrachloride & ${ }^{35} \mathrm{Cl}$ & $\begin{array}{l}40.6 \\
(77 \mathrm{~K})\end{array}$ & - & 0.1\|\|$^{x}$ \\
\hline \multirow[t]{2}{*}{ Boron trichloride } & ${ }^{35} \mathrm{Cl}(\mathrm{I})$ & $\begin{array}{l}21.5822 \\
(77 \mathrm{~K})\end{array}$ & - & $\begin{array}{l}0.5^{*}+\dagger \\
0.54^{* *, y}\end{array}$ \\
\hline & ${ }^{35} \mathrm{Cl}(\mathrm{II})$ & $\begin{array}{l}21.5785 \\
(77 \mathrm{~K})\end{array}$ & - & $0.47^{*, \dagger \dagger}$ \\
\hline \multirow[t]{2}{*}{ Phenylboron dichloride } & ${ }^{35} \mathrm{Cl}(\mathrm{I})$ & $\begin{array}{l}20.096 \\
(77 \mathrm{~K})\end{array}$ & - & $0.57^{* *, y}$ \\
\hline & ${ }^{35} \mathrm{Cl}(\mathrm{II})$ & $\begin{array}{l}20.226 \\
(77 \mathrm{~K})\end{array}$ & - & \\
\hline \multirow[t]{2}{*}{ Chloroform } & ${ }^{35} \mathrm{Cl}(\mathrm{I})$ & $\begin{array}{l}38.2553 \\
(77 \mathrm{~K})\end{array}$ & - & $0.29 \|^{e}$ \\
\hline & ${ }^{35} \mathrm{Cl}(\mathrm{II})$ & $\begin{array}{l}38.3085 \\
(77 \mathrm{~K})\end{array}$ & - & $0.21 \|$ \\
\hline Methylene chloride & ${ }^{35} \mathrm{Cl}$ & $\begin{array}{l}35.9912 \\
(77 \mathrm{~K})\end{array}$ & - & $0.43 \|^{, e}$ \\
\hline \multirow[t]{2}{*}{ Deuterochloroform } & ${ }^{35} \mathrm{Cl}(\mathrm{I})$ & $\begin{array}{l}38.2626 \\
(77 \mathrm{~K})\end{array}$ & - & $0.31 \|^{e}$ \\
\hline & ${ }^{35} \mathrm{Cl}(\mathrm{II})$ & $\begin{array}{l}38.3125 \\
(77 \mathrm{~K})\end{array}$ & - & $0.19 \|$ \\
\hline \multirow[t]{2}{*}{ Trichloroethane } & ${ }^{35} \mathrm{Cl}(\mathrm{I})$ & $\begin{array}{l}37.8352 \\
(77 \mathrm{~K})\end{array}$ & - & $0.29 \|^{e}$ \\
\hline & ${ }^{35} \mathrm{Cl}(\mathrm{II})$ & 38.0461 & - & $0.19 \|$ \\
\hline Transdichloroethylene & ${ }^{35} \mathrm{Cl}$ & $\begin{array}{l}35.480 \\
(83 \mathrm{~K})\end{array}$ & - & $0.265^{*, g}$ \\
\hline Chlorobenzene & ${ }^{35} \mathrm{Cl}$ & $\begin{array}{l}34.6224 \\
(77 \mathrm{~K})\end{array}$ & - & $0.10 \|, z$ \\
\hline
\end{tabular}

*Externally quenched super-regenerative spectrometer was used in the measurements.

**Externally quenched super-regenerative spectrometer with Zeeman modulation was used in the measurements.

IInternally quenched super-regenerative spectrometer was used in the measurements.

$\S R$ egenerative spectrometer was used in the analysis.

$\dagger$ Frequency-field method was used for the analysis.

$\dagger \dagger$ Present work.

${ }^{a}$ T. Okuda, A. Nakao, M. Shiroyama and H. Negita, Bull. Chem. Soc. Japan 41, 61 (1968).

${ }^{b} \mathrm{G}$. E. Peterson, Thesis, University of Pittsburgh (1962).

${ }^{\prime}$ K. V. Raman and P. T. Narasimhan (to be published).

${ }^{d}$ G. E. Peterson and P. M. Bridenbaugh, J. Chem. Phys. 51, 238 (1969).

${ }^{e}$ Dinesh, K. V. Raman and P. T. Narasimhan, paper presented at the Third International Conference on Magnetic Resonance at Australia, August (1969).

${ }^{\delta}$ S. Chu, G. A. Jeffrey and T. Sakurai, Acta. Cryst. 15, 661 (1962).

A. K. Saha, R. Roy and S. Sengupta, Proceedings of Solid State and Nuclear Physics Symposium, Bombay, India, (December 1968).

${ }^{h}$ C. Dean, Phys. Rev. 86, 607 (1952).

iS. Ogawa and K. Ohi, J. Phys. Soc. Japan, 15, 1064 (1960).

${ }^{k}$ V. Rehn, J. Chem. Phys. 38, 749 (1963).

'P. Bucci, P. Cecchi and E. Scrocco, Ric. Sci. 34, 129 (1964).

${ }^{m}$ Y. Morino and M. Toyama, J. Chem. Phys. 35, 1289 (1961).

"Y. Morino, T. Chiba, T. Shimozawa and M. Toyama, J. Phys. Soc. Japan 13, 869 (1958)

${ }^{P}$ F. J. Adrian, J. Chem. Phys. 29, 1381 (1958).

'F. J. Adrian, J. Chem. Phys. 38, 1258 (1963).

${ }^{s}$ E. A. C. Lucken and C. Mazeline, J. Chem. Soc. A 153 (1968).

${ }^{\prime}$ H. Negita, T. Tanaka, T. Okuda and H. Shimada, Inorg. Chem. 5, 2126 (1966).

"J. Ramakrishna, Phil. Mag. 14, 589 (1966).

${ }^{\circ}$ Dinesh and P. T. Narasimhan, J. Chem. Phys. 45, 2170 (1966).

JJ. D. Graybeal and P. J. Green, J. Phys. Chem. 73, 2948 (1969).

'J. A. S. Smith and D. A. Tong, J. Chem. Soc. A $173(1971)$.

'Dinesh and P. T. Narasimhan, J. Chem. Phys. 49, 2519 (1968). 
quenched super-regenerative spectrometers can be avoided by using externally quenched spectrometers and side-band suppression ${ }^{12}$. Signal enhancements can be also achieved using magnetic field modulation ${ }^{13}$. The accuracy of the $\eta$ values obtained from powder specimens is not high compared to the single crystal data especially using the frequency-field method. Further, information on the orientation of the electric field gradients is not available from the powder measurements. In spite of these drawbacks there is a great advantage to this method in that it does not demand the use of single crystals and is therefore especially valuable when substances with low melting points are to be studied. Further, the design and construction of sophisticated goniometer assemblies is avoided by this method.

\section{COMPARISON OF $\eta$ MEASUREMENTS ON SINGLE CRYSTALS AND POWDER SAMPLES}

We shall now compare the results obtained by using powder specimens and single crystal specimens. We have also taken this opportunity to collect all available powder data. Table 1 gives the results on asymmetry parameter measurements for spin $\frac{2}{2}$ systems, namely, ${ }^{35} \mathrm{Cl}$ and ${ }^{71} \mathrm{Ga}$ using polycrystalline specimens. Other nuclei do not appear to have been studied by the powder method. We are at present engaged in extending our studies in this direction. The single crystal data are also presented in the Table for comparison, wherever available. It is surprising to note that a total of only six systems involving two nuclei, namely ${ }^{35} \mathrm{Cl}$ and ${ }^{71} \mathrm{Ga}$, have been studied by the powder method for which single crystal data are also available. Out of these, in the case of mercuric chloride and gallium chloride the powder $\eta$ values are quite different from the values obtained from single crystal specimens. In others where the single crystal data are available both the methods agree well. It is gratifying to note that the powder results on $p$-dichlorobenzene and boron trichloride carried out by independent groups of workers agree well.

\section{THE UTILITY OF COMPUTER SIMULATED ZEEMAN NQR SPECTRA FOR POWDER SPECIMENS}

A computer simulation of the theoretical Zeeman spectrum using Gaussian line shapes is especially useful for systems like boron trichloride which have very closely spaced pure nqr lines. Theoretical powder Zeeman spectra were earlier computed by Adrian ${ }^{14}$ and later by Graybeal and Green ${ }^{15}$ for some chlorine compounds. Following the work of Adrian we illustrate here the usefulness of the computer simulated spectra in the case of boron trichloride and $p$-dichlorobenzene. The ${ }^{35} \mathrm{Cl}$ nqr spectrum of boron trichloride consists of a closely-spaced doublet line (Figure $3 a$ ). The two nqr lines arise due to the isotope effect of ${ }^{10} \mathrm{~B}$ and ${ }^{11} \mathrm{~B}$ as discussed earlier by Douglass ${ }^{16}$ and $\mathrm{Chiba}^{17}$. The integrated intensity of the two lines in the doublet is expected to be in the ratio of the isotopic abundance of ${ }^{10} \mathrm{~B}$ and ${ }^{11} \mathrm{~B}$, namely $18.83: 81.17$. This is also in agreement with experiment. We first simulated on the computer the theoretical pure quadrupole spectrum of the doublet in the absence of the magnetic field by mixing two Gaussian lines with the derivative line shape function given by the expression 


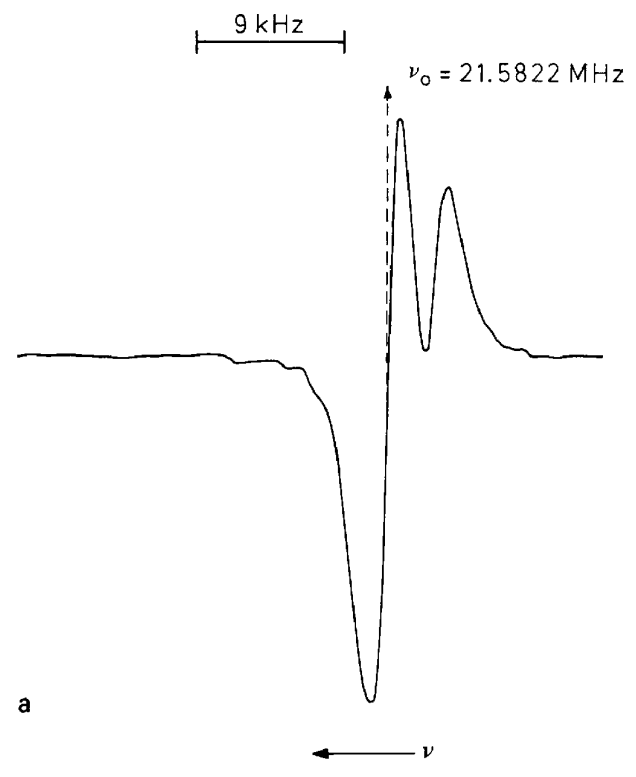

Figure $3 a$. The pure nqr spectrum of ${ }^{35} \mathrm{Cl}$ in boron trichloride observed experimentally at $77 \mathrm{~K}$.

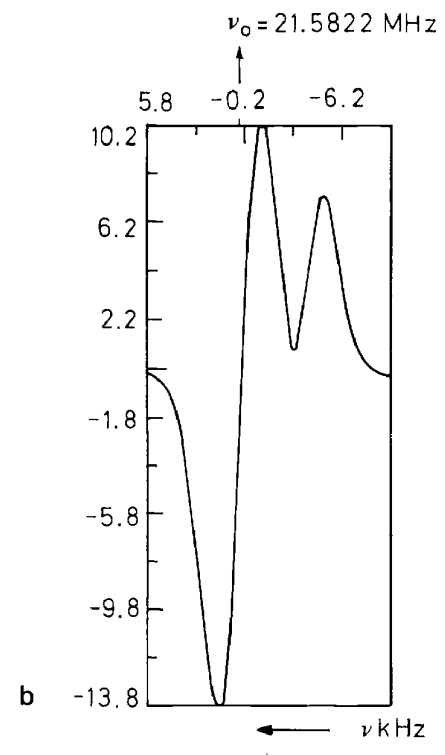

$b$. Theoretical (computer simulated) nqr spectrum of ${ }^{35} \mathrm{Cl}$ in boron trichloride. 


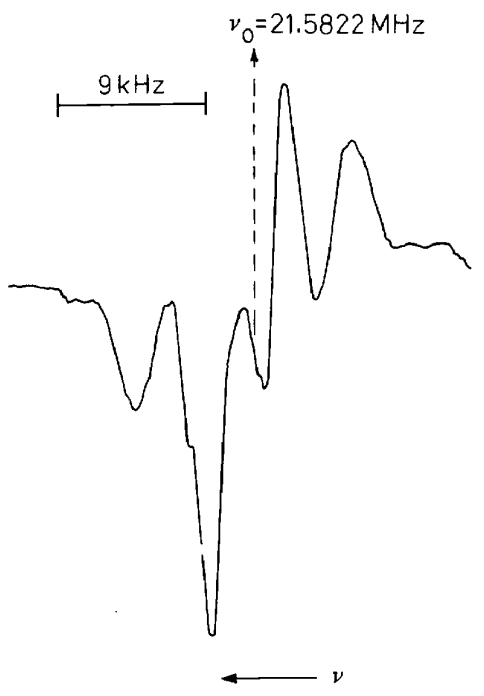

Figure $4 a$. The Zeeman nqr spectrum of ${ }^{35} \mathrm{Cl}$ in boron trichloride observed experimentally at 10.422 gauss at $77 \mathrm{~K} . b$. The theoretical Zeeman nqr spectrum of the separated nqr lines at $H=10.442$ gauss in boron trichloride $\left(\eta_{C \mid(I)}=0.5, \eta_{C \mid(I)}=0.47\right)$ (see Table 1). $c$. The Zeeman nqr spectrum of ${ }^{35} \mathrm{Cl}$ in boron trichloride theoretically simulated at 10.442 gauss using $\eta_{1}=0.5$, $\eta_{2}=0.47$ where $\eta_{1}$ and $\eta_{2}$ are the $\eta$ values used for the $21.5822 \mathrm{MHz}$ and $21.5785 \mathrm{MHz}$ lines respectively. $d$. The Zeeman nqr spectrum of ${ }^{35} \mathrm{Cl}$ in boron trichloride theoretically simulated at 10.442 gauss using $\eta_{1}=\eta_{2}=0.3$. e. The Zeeman nqr spectrum of ${ }^{35} \mathrm{Cl}$ in boron trichloride theoretically simulated at 10.442 gauss using $\eta_{1}=\eta_{2}=0.6$.

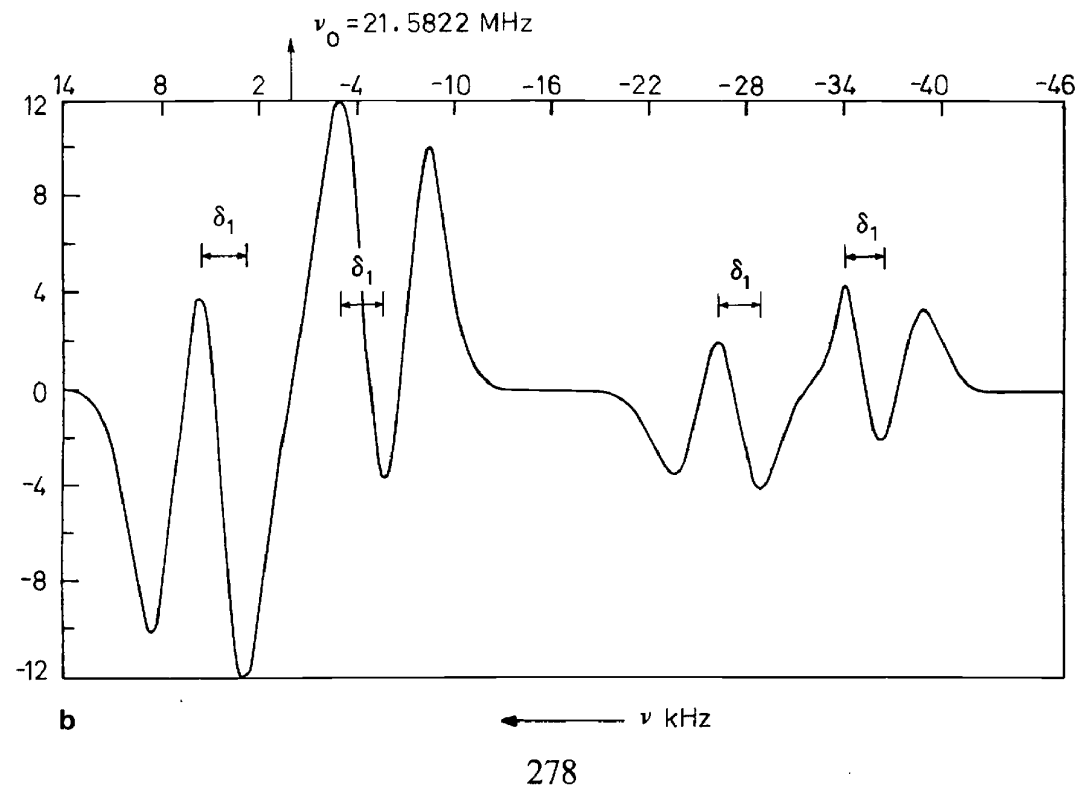


ASYMMETRY PARAMETERS FOR SPIN 3/2 NUCLEI

$v_{0}=21.5822 \mathrm{MHz}$
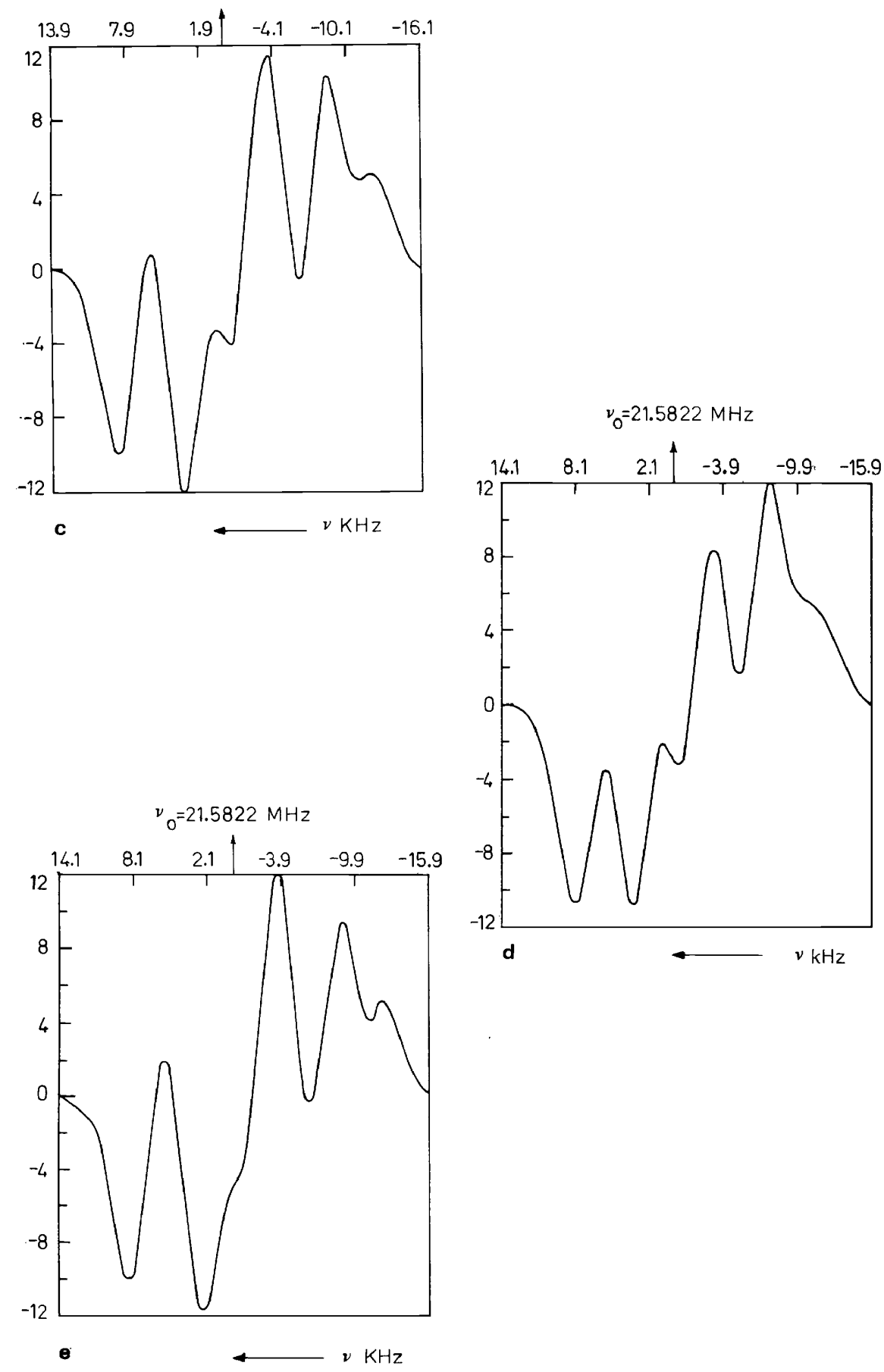


$$
\begin{gathered}
\frac{\mathrm{d}}{\mathrm{d} v_{r}} g\left(v_{r}\right)=\left(\frac{32}{\pi}\right)^{\frac{1}{2}} \frac{1}{\sigma_{r}^{2}} \frac{v-v_{0_{r}}}{\sigma_{r}} \exp \left\{-2\left(\frac{v-v_{0_{r}}}{\sigma_{r}}\right)^{2}\right\} \\
(r=1,2)
\end{gathered}
$$

Here $\sigma_{r}$ is the linewidth of the $r$ th nqr line without the magnetic field. The integrated intensity ratio of the two 'separated' lines was then demanded to satisfy the ratio of the natural abundances of the two boron isotopes. By choosing line widths for the $21.5822 \mathrm{MHz}$ and $21.5785 \mathrm{MHz}$ lines as 3.0 and $2.5 \mathrm{kHz}$ respectively and using the value of $3.68 \mathrm{kHz}$ reported by $\mathrm{Chiba}^{17}$ for the separation between them, the best fit between experimental and theoretical nqr spectra was obtained. Figure $3 b$ shows the theoretical nqr spectrum. Following the work of Adrian ${ }^{14}$ the theoretical Zeeman nqr spectrum at different fields can be obtained using the derivative form for the envelope shape function where the envelope shape function is given by the expression

$$
\begin{aligned}
& I\left(v_{r}\right)=\sum_{i=\alpha, \alpha^{\prime}, \beta, \beta^{\prime} .} \int_{0}^{\pi / 2} \sin \theta \mathrm{d} \theta \int_{0}^{\pi / 2} \mathrm{~d} \phi I_{r_{i}}^{\prime}(\theta, \phi) \\
& \times \exp \left\{-2 \frac{\left(v-v_{r_{i}}^{\prime}(\theta, \phi)\right)^{2}}{\sigma_{r}^{2}}\right\} \\
&(r=1,2)
\end{aligned}
$$

$\sigma_{r}$ is the linewidth of the nqr line in the absence of a magnetic field and $v_{r_{i}}(\theta, \phi)$ and $I_{r_{i}}^{\prime}(\theta . \phi)$ are frequency and intensity of the $i$ th component of the $r$ th line and $\theta, \phi$ are the polar and azimuthal angles specifying the orientation of the external magnetic field with respect to the principal axes of the electric field gradient. The best fit between theoretical and experimental Zeeman spectra was obtained for $\eta$ values of 0.5 and 0.47 for the $21.5822 \mathrm{MHz}$ line and $21.5785 \mathrm{MHz}$ line respectively. Figure $4 a$ shows the ${ }^{35} \mathrm{Cl}$ Zeeman nqr spectrum observed experimentally at $H=10.422$ gauss. Figure $4 b$ shows the theoretical Zeeman nqr spectrum of the separated nqr lines at $H=10.442$ gauss. Figure $4 c$ shows the theoretical spectrum obtained by combining appropriately the two parts of the spectrum shown in Figure $4 b$. Figures $4 d$ and $4 e$ show the computer simulated Zeeman spectra at $H=10.442$ gauss for different sets of $\eta$ values. The simulated spectrum with the values $\eta=0.5$ for one site and $\eta=0.47$ for the other site is in close agreement with experiment. The Zeeman spectra at other fields were then simulated using this set of $\eta$ values and the spectra compared with experiment. The overall agreement was thus established. In an independent study of boron trichloride Smith and Tong ${ }^{13}$ assumed the ${ }^{35} \mathrm{Cl}$ line to be a single line and analyzed the Zeeman pattern using the powder method. They obtained an average value of $\eta=0.54$.

We now come to the case of $p$-dichlorobenzene. Figure $5 a$ and Figure $5 b$ show the theoretically simulated spectra for the ${ }^{35} \mathrm{Cl}$ resonance using different $\eta$ values at $H=16.2$ gauss. With a value of $\eta=0.07$ we can reproduce the experimental spectrum of Morino and Toyama ${ }^{3}$. The agreement with their value is seen to be good. We have also examined the effect of introducing the 'background' line on the Zeeman spectra. Although the $\eta$-peaks 


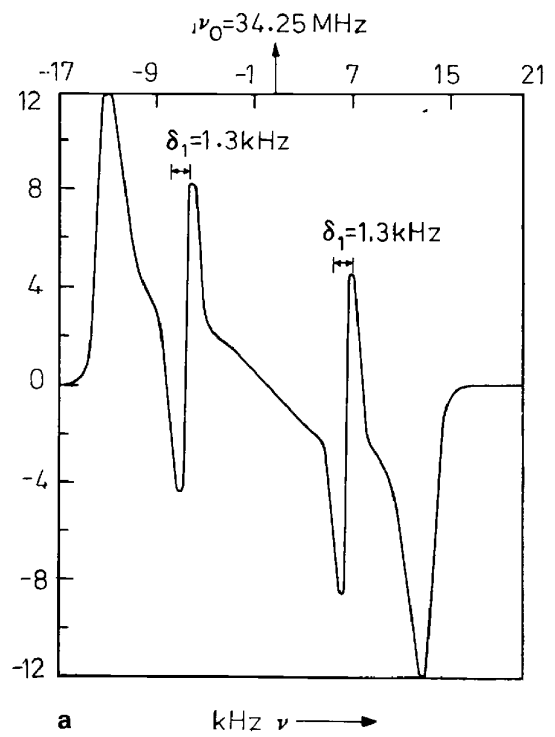

Figure 5a. The theoretical Zeeman nqr spectrum of ${ }^{35} \mathrm{Cl}$ site in $p$-dichlorobenzene at $H=16.2$ gauss with $\eta=0.07$. $b$. The theoretical Zeeman nqr spectrum of ${ }^{35} \mathrm{Cl}$ site in $p$-dichlorobenzene at $H=16.2$ gauss with $\eta=0.14$.

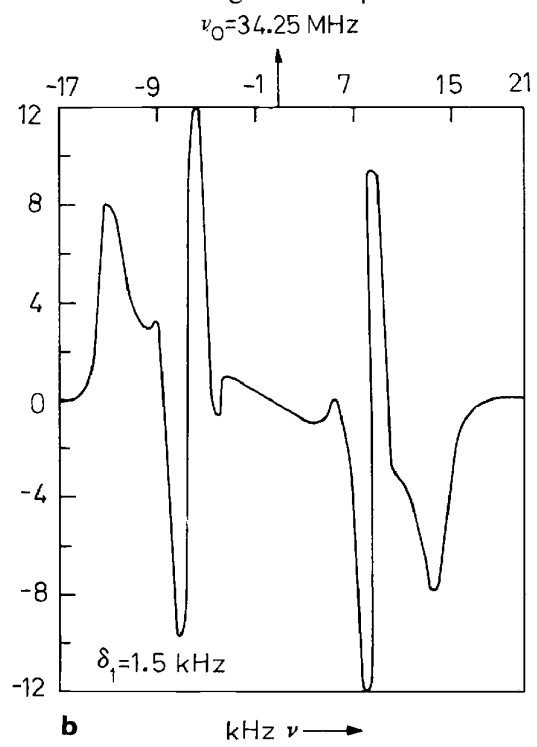

get shifted slightly with the introduction of the background line the error thus introduced in the measurement of the $\eta$-peaks is not serious. The line shape of course is affected by the intensity of the background line. One of the merits of the computer simulation method is that it de-emphasizes the extrapolation of linewidth data with respect to field. 


\section{FACTORS THAT MIGHT CONTRIBUTE TO THE DIFFERENCES IN $\eta$ VALUES OBTAINED USING THE POWDER METHOD AND SINGLE CRYSTAL SPECIMENS}

As noted earlier the results on $\eta$ measurements obtained by the powder method and the single crystal method agree well except in the cases of mercuric chloride and gallium trichloride. $\eta$ values are available by the powder method for ${ }^{35} \mathrm{Cl}$ and ${ }^{71} \mathrm{Ga}$ sites. In the case of gallium trichloride, except for one chlorine site, all other $\eta$ values obtained from powder specimens are different from the single crystal data of Peterson and Bridenbaugh ${ }^{5 b}$. We have carried out a theoretical computer simulation of the gallium trichloride powder Zeeman spectra for the ${ }^{35} \mathrm{Cl}$ and ${ }^{71} \mathrm{Ga}$ sites and we find that the agreement between experimental splittings and theoretical splittings is good using our powder $\eta$ values. Figure $6 a$ and $6 b$ illustrate this situation. On the other hand, when we used $\eta$ values from single crystal data and simulated the spectra we found that these were not in good agreement with the powder spectra. Figure $6 c$ gives the ${ }^{71} \mathrm{Ga}$ spectrum obtained by computer simulation using single crystal data and it can be clearly seen that the single crystal value does not yield good agreement with the powder Zeeman spectrum. In the case of mercuric chloride, we have attempted to simulate the experimental powder Zeeman spectrum obtained with an externally quenched superregenerative spectrometer and find that $\eta$ values $(0.45-0.48)$, higher than those reported by us earlier ${ }^{4 a}$, are needed to obtain good agreement with experiment. The computer simulated spectrum with low $\eta$ values does not match well with the experimental powder Zeeman pattern (see Figure $7 a, b$ and $c$ ). It is thus clear that the powder results are at variance with the single crystal data here also. It is therefore worthwhile to consider in detail some of the factors that might contribute to the difference between the powder and single crystal values. The theory as developed by Morino and Toyama for the nonaxial case is clearly applicable only to first order in $\eta$. For large $\eta$ values therefore the theory is approximate, if not invalid. However at least in one

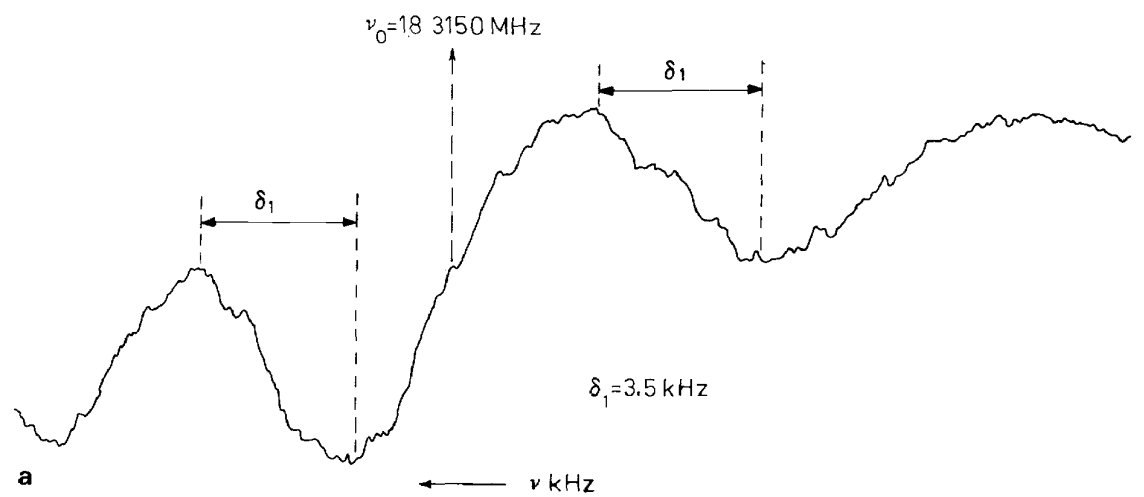

Figure $6 a$. The experimental Zeeman nqr spectrum of ${ }^{71} \mathrm{Ga}$ site in gallium trichloride at $H=$ 5.25 gauss. $b$. The theoretical Zeeman nqr spectrum of ${ }^{71} \mathrm{Ga}$ site in gallium trichloride at $H=$ 5.25 gauss with $\eta=0.26$ (powder value). $c$. The theoretical Zeeman nqr spectrum of ${ }^{71} \mathrm{Ga}$ site in gallium trichloride at $H=5.25$ gauss with $\eta=0.867$ (single crystal value). 


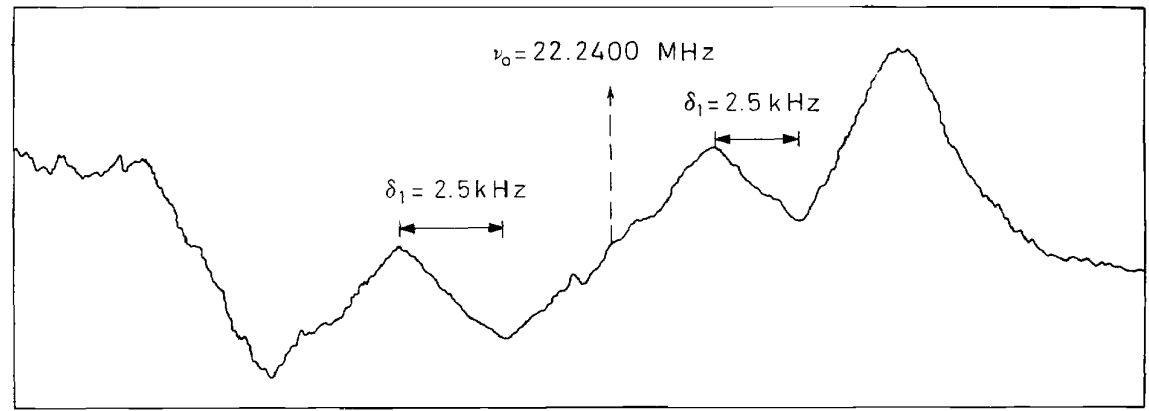

a

$k H z v$

Figure 7a. The experimental Zeeman NQR spectrum of ${ }^{35} \mathrm{Cl}$ in mercuric chloride at $H=9.48$ gauss. $b$. The theoretical Zeeman NQR spectrum of ${ }^{35} \mathrm{Cl}$ in mercuric chloride at $H=9.48$ gauss with $\eta=0.47$. c. The theoretical Zeeman NQR spectrum of ${ }^{35} \mathrm{Cl}$ in mercuric chloride at $H=9.48$ gauss with $\eta=0.08$ (single crystal value).

\section{$\nu_{0}=22.2400 \mathrm{MHz}$}

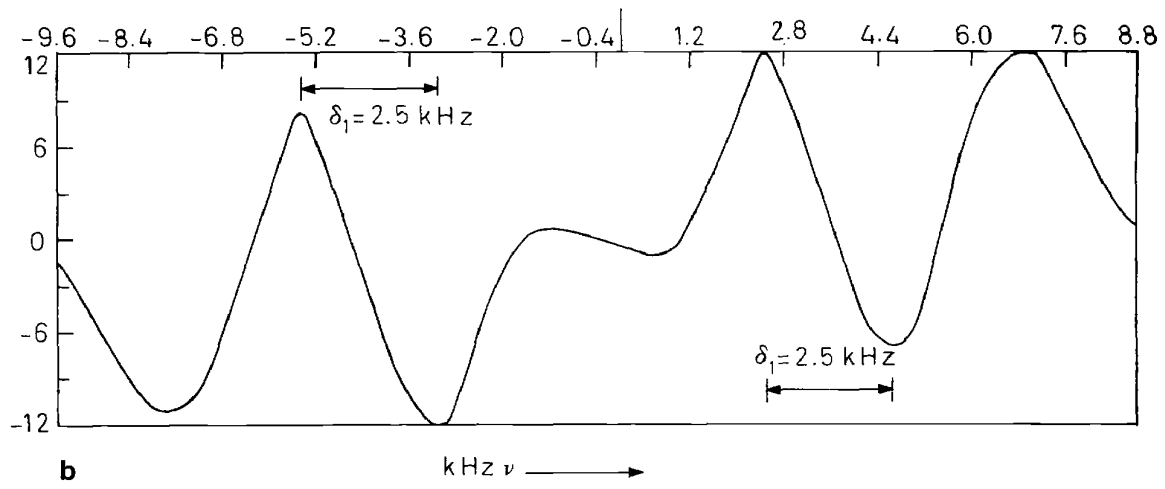

$\nu_{0}=22.2400 \mathrm{MHz}$

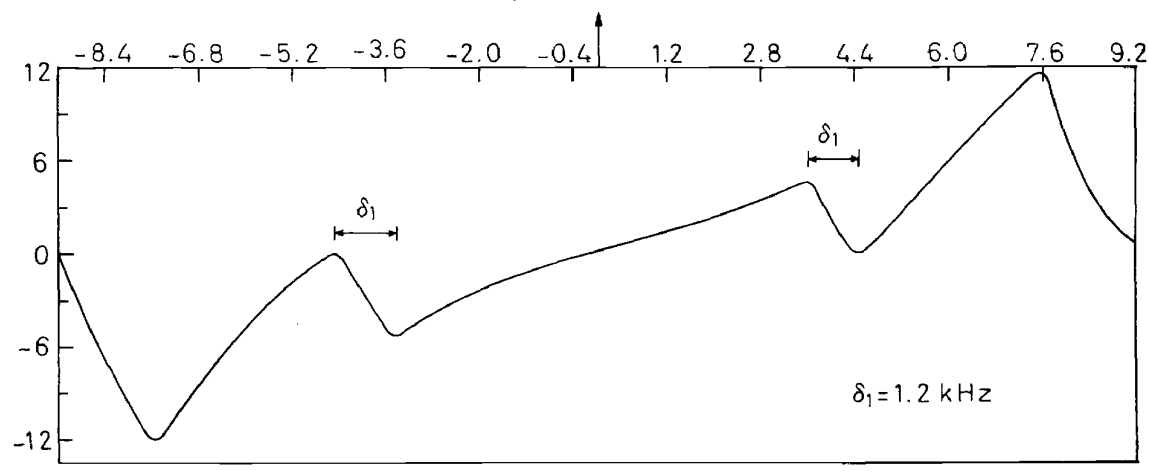

c

$k H z v$ 
that Adrian in his measurement of the asymmetry parameter of chlorine molecule had to rely on the computer simulated spectra heavily and further could only fix the upper limit of $\eta(0.03)$. When one is not forced into such experimental difficulties in the powder method the recording of powder Zeeman pattern can be done with fairly good $S / N$ ratios and as has been shown in the previous section the observed Zeeman spectra in some cases can only be reconciled with $\eta$ values which are quite different from those obtained by single crystal data. The uncertainty in the choice of $\delta$ values, namely $\delta_{1}, \delta_{2}, \delta_{3}$ for extrapolating to infinite field, is another point that has to be borne in mind in judging the powder data ${ }^{18,19}$. Although computer simulation of the spectrum can be helpful it is necessary to extend the experimental data to higher fields. In doing so one faces the problem of establishing correspondence between the theoretically-simulated computer spectra and experimental spectra taken under adverse conditions. The Morino-Toyama theory demands random orientation of the polycrystalline material. While in many organic compounds it is possible to meet this requirement by sudden freezing of the gas or liquid sample it may not be the case in a few situations.

Single crystals which are grown under different conditions may exhibit varying degrees of strain and in the polycrystalline sample strain does not pose problems. Yet another possibility that has been suggested ${ }^{20}$ is the role of chemical shift anisotropy. In other words, in the case of the single crystal method the results of measurements may be subject to a directional dependence on the magnetic field which is not wholly due to the quadrupole Hamiltonian. The $\eta$-results will then be affected by the chemical shift anisotropy and the orientation of the chemical shift tensor. There is therefore a need to extend Zeeman investigations of spin $I=\frac{3}{2}$ nuclei in both powder and single crystal forms and to obtain data on chemical shift anisotropy as well.

\section{SUMMARY}

A survey of the available powder measurements on spin $I=\frac{3}{2}$ nuclei has been made. In the analysis of powder data it has been shown that computer simulation of nqr Zeeman spectra can be valuable. On comparing the powder data with single crystal data wherever available it is found that there are certain discrepancies between the powder and single crystal values. The possible factors that may contribute to these differences have been examined.

\section{ACKNOWLEDGEMENT}

One of us (KVR) wishes to acknowledge with grateful thanks the award of a Junior Research Fellowship from the Department of Atomic Energy, Government of India. Our thanks are due to the Staff of the Computer Centre, IITK for their valuable cooperation. PTN wishes to thank the organizers of the Fourth International Symposium on Magnetic Resonance and Prof. D. Fiat in particular for the kind invitation to present this paper and extending a warm hospitality. 


\section{REFERENCES}

${ }^{1}$ C. Dean, Phys. Rev. 86, 607 (1952).

2 V. Rehn, J. Chem. Phys. 38, 749 (1963).

3 Y. Morino and M. Toyama, J. Chem. Phys. 35, 1289 (1961).

4 (a) Dinesh and P. T. Narasimhan, J. Chem. Phys. 45, 2170 (1966).

(b) H. Negita, T. Tanaka, T. Okuda and H. Shimada, Inorg. Chem. 5, 2126 (1966).

(c) J. Ramakrishna, Phil. Mag. 14, 589 (1966).

5 (a) Dinesh, K. V. Raman and P. T. Narasimhan, Paper presented at the Third International Conference on Magnetic Resonance at Australia, August (1969).

(b) G. E. Peterson and P. M. Bridenbaugh, J. Chem. Phys. 51, 238 (1969).

6 P. Bucci, P. Cecchi and A. Colligiani, J. Chem. Phys. 50, 530 (1969).

7 Y. Morino, T. Chiba, T. Shimozawa and M. Toyama, J. Phys. Soc. Japan, 13, 869 (1958).

${ }^{8}$ G. E. Peterson and P. M. Bridenbaugh. Rev. Sci. Inst. 38. 387 (1967).

9 A. K. Saha, S. Sengupta and R. Roy, Proceedings of Symp. Nucl. Phys. Solid State Phys. page 312 , Madras, India (1968).

10 J. A. S. Smith and D. A. Tong, J. Sci. Inst. (J. Phys. E) Series 21,8 (1968).

11 C. Dean, Rev. Sci. Inst. 31, 934 (1960).

12 C. Dean and M. Pollak, Rev. Sci. Inst. 29, 630 (1958).

13 J. A. S. Smith and D. A. Tong, J. Chem. Soc. A, 173 (1971).

14 F. J. Adrian, J. Chem. Phys. 38, 1258 (1963).

15 J. D. Graybeal and P. J. Green, J. Phys. Chem. 73, 2948 (1969).

16 D. C. Douglas, Ph.D. Thesis, Cornell University (1957).

17 T. Chiba, J. Phys. Soc. Japan 13, 860 (1958).

18 Dinesh and P. T. Narasimhan, J. Chem. Phis. 49. 2519 (1968).

${ }^{19}$ P. T. Narasimhan, Proceedings of Symp. Nucl. Solid State Phys. page 317, Madras, India (1968).

${ }^{20}$ E. A. C. Lucken, Nuclear Quadrupole Coupling Constants, page 308, Academic Press, London (1969). 